\title{
Mechanisms of Drug Desensitization: Not Only Mast Cells
}

\author{
Alessandra Vultaggio ${ }^{1 *}$, Andrea Matucci ${ }^{1}$, Francesca Nencini ${ }^{1}$, Susanna Bormioli ${ }^{2}$, \\ Emanuele Vivarelli ${ }^{1}$ and Enrico Maggi ${ }^{3}$ \\ ${ }^{1}$ Immunoallergology Unit, Careggi University Hospital, Florence, Italy, ${ }^{2}$ Immunology and Cellular Therapy, Careggi University \\ Hospital, Florence, Italy, ${ }^{3}$ Translational Immunology Unit, Immunology Area, Pediatric Hospital Bambino Gesù, IRCCS, Rome, \\ Italy
}

Drug desensitization (DD) allows transient clinical tolerance to the drug in reactive patients and it is frequently and successfully used in the management of both IgE and non lgEmediated hypersensitivity reactions (HRs). The underlying mechanisms behind this process is not well understood. The desensitization procedure is associated with the inhibition of mast cells degranulation and cytokine production, that, is attributable, at least partially, to the abrogation of Ca2+ mobilization; in vitro findings and in vivo mouse models of rapid desensitization show that the organization and spatial distribution of actin is critical for $\mathrm{Ca} 2+$ mobilization. Some clinical observations may suggest the induction of a longer memory of tolerance by DD and they raise the suspicion that other cells and mechanisms are involved in DD. Some data are emerging about the modifications of immune responses

OPEN ACCESS

Edited by: Maria Jose Torres, University of Málaga, Spain

Reviewed by:

Vito Sabato,

University of Antwerp, Belgium

Rui Liu,

Xi'an Jiaotong University, China

*Correspondence: Alessandra Vultaggio vultaggioalessandra@gmail.com

Specialty section:

This article was submitted to Translational Pharmacology, a section of the journal

Frontiers in Pharmacology

Received: 03 August 2020 Accepted: 25 November 2020 Published: 23 December 2020

Citation: Vultaggio A, Matucci A, Nencini F, Bormioli S, Vivarelli $E$ and Maggi $E$ (2020) Mechanisms of Drug Desensitization: Not Only Mast Cells.

Front. Pharmacol. 11:590991. doi: 10.3389/fphar.2020.590991 during DD in patients with previous immediate HRs. In particular, an increase of regulatory cytokines, mainly represented by $\mathrm{IL}-10$, has been shown, and more importantly, the appearance of IL-35 producing $T$ regulatory cells has been described during DD. The release of controlled cellular mediators by mast cells over time and the development of the antigen-specific regulation of adaptive response allow to safely and successfully reach the target dose of a first line drug during DD.

Keywords: desensetisation, allergy (hypersensitive anaphylaxis), mast cells, T reg cell, IL-10, IL-35

\section{INTRODUCTION}

The treatment of many disorders including cancer and autoimmune diseases can be complicated by hypersensitivity reactions (HRs). Clinical manifestations vary considerably, ranging from mild to severe and life-threatening reactions leading to drug discontinuation, which in turn can decrease patients' quality of life and/or life expectancy (Vultaggio et al., 2011; Sala-Cunill et al., 2019). Phenotypes in drug allergy focus on symptoms and timing, classifying the reactions as immediate or delayed, depending on the time between treatment administration and the onset of symptoms (Bonamichi-Santos and Castells, 2016). The most frequently involved culprit drugs are represented by antibiotics, aspirin, chemotherapeutics (mainly platinum compounds and taxanes) and biological agents (Vultaggio and Castells, 2014).

Management of HRs, beyond an allergological work-up aimed to define the pathogenic mechanism of the reaction, may include drug desensitization (DD) when there is no alternative therapy available. The culprit drug is usually avoided in order to prevent future reactions and DD was developed as a treatment option to maintain patients on first line therapy (Castells, 2017).

The activation of mast cells (MC) plays a critical role in HRs, not only limited to the immediate release of an array of preformed inflammatory mediators including histamine, tryptase, serotonin, 
chymases, cytokines, and growth factors, but also de novo synthesis of lipid mediators such as leukotrienes. In addition to the classical IgE-mediated MC activation, other mechanisms may be involved. Some medications can directly activate MC via the recently identified Mas-related G Protein Coupled Receptor-X2 (MRGPRX2) transmembrane protein, as in the case of fluoroquinolones, neuromuscular blocking agents, and vancomycin (McNeil et al., 2015; Boyce, 2019). Others may further activate mast cells via complement activation leading to the production of anaphylotoxins C3a and C5a (Jimenez-Rodriguez et al., 2018). The direct activation of membrane receptors as in the case of opioids and estrogens, represent a non-immunological pathway of MC activation possibly involved in the induction of HRs (Spoerl et al., 2017). Finally, cyclooxygenase-1 inhibition [as in the case of Aspirin Exacerbated Respiratory disease (AERD)] may occur.

The aim of this review is to evaluate the mechanisms involved in successuful DD, highlighting the role of regulatory cells and cytokines in the modulation of a drug-specific immune response.

\section{DRUG DESENSITIZATION: GENERAL CONCEPTS}

Management of HRs in patients without treatment alternatives is based on the DD procedure, able to induce a temporary hyporesponsive state by incremental escalation of sub-optimal doses of the offending drug, until reaching required dosage (De las Vecillas Sanchez et al., 2017). Drug desensitization was developed due to the pressing need to reintroduce drugs in a safe fashion in patients who had developed both IgE-and/or non IgE-mediated HRs to critical drugs. Because DD is able to induce a temporary tolerance to the culprit drug, and considering that some medications (chemotherapy, biologic agents) have prolonged dosing intervals, subsequent administrations must be preceded by a DD procedure in order to overcome the loss of tolerance.

Desensitization is conceptually dedicated to patients in which an IgE-mediated mechanism is demonstrated by positive skin testing or serum IgE for culprit drug, however, patients who suffered immediate reactions to taxanes and other chemotherapies in which the IgE mechanisms cannot be demonstrated have also been successfully desensitized (Madrigal-Burgaleta et al., 2019).

Two types of DD protocols are available: rapid drug desensitization which addresses type I reactions with mast cells/basophils/IgE involvement, and slow drug desensitization which addresses delayed type IV reactions with T-cell involvement (Castells, 2015). Mixed reactions however have become more frequent, so DD protocols have slowly changed the segregation paradigm of DD vs. slow drug desensitization (Pyle et al., 2014). Desensitization is contraindicated in patients whose reaction suggests a history of severe cutaneous reactions, such as Stevens-Johnsons syndrome, toxic epidermal necrolysis, drug induced hypersensitivity syndrome, drug reaction (rash) with eosinophilia and systemic symptoms and acute generalized exanthematous pustulosis. Desensitization is also not considered appropriate for reactions of serum sickness or haemolytic anemia (Castells, 2017). Omalizumab is a humanized IgG1 monoclonal antibody, initially approved for the treatment of severe allergic asthma and more recently, for the treatment of chronic idiopathic urticaria. In several case reports it has been applied to control the reactions occurring during $\mathrm{DD}$, for aspirin (Waldram et al., 2018), insulin (Mishra et al., 2018), elosulfasi a (Guvenir et al., 2017), carboplatin (Oude Elberink et al., 2020), and oxaliplatin (Prieto-Garcia et al., 2019).

\section{MECHANISMS INVOLVED IN DRUG DESENSITIZATION: MAST CELLS}

Regardless of whether the reaction is the consequence of an IgEor non IgE-mediated mechanism, MC are key effector cells in the majority of immediate drug reactions, and the desensitization procedure is associated with the inhibition of MC degranulation and cytokine production.

Both in vivo and in vitro studies have been used to understand the cellular and molecular pathways influencing the function of MC and basophils during DD.

Several observations displayed negative skin testing after desensitization, indicating inhibition of the MC activation. These data have been extensively described in DD for chemotherapeutics and more recently for biological agents (Lee et al., 2004). By using sensitized bone marrow-derived MC under physiologic calcium conditions and by administrating incremental doses of the drug at fixed time intervals, cells were shown to become unresponsive (SanchoSerra et al., 2011). Recent data obtained in a subject sensitized to infliximab (IFX) and grass pollen, who experienced an immediate HR to IFX, showed that skin testing for IFX was positive before cycles of DD but negativized after each procedure, while skin testing for grass pollen remained positive before and after each cycle (Vultaggio et al., 2020). These data obtained in humans were confirmed by in vitro results. In fact, challenging with the culprit drug after being desensitized did not induce in vitro activation of MC that could still be activated by different antigen stimulation, supporting the concept that DD is an antigen-specific process (Gladys et al., 2016).

To understand the mechanisms by which DD procedures impact MC mediator release, it is useful to define sequential events starting from IgE/FceRI cross-linking to the intracellular signals.

Phosphorylation of subunit ITAMs (immune-receptor tyrosine-based activation motif) is important in initiating and inducing downstream propagation of intracellular signaling (Phong et al., 2015). Activated Lyn initiates signal transduction through phosphorylation of the $\beta$ and $\gamma$ ITAM chain. In the first phase of the process, phospholipase $\mathrm{C} \gamma$ phosphorylates and then hydrolyzes phosphatidyl inositol bisphosphate to yield inositol trisphosphate (IP3) and diacylglycerol (DAG). IP3 induces an increase in cytosolic calcium ion $(\mathrm{Ca} 2+)$ concentration, by binding to its receptor in the endoplasmic reticulum and rapidly inducing the process of 
calcium mobilization. In the subsequent phase, a prolonged $\mathrm{Ca} 2+$ influx occurs (Nam and Kim, 2020).

Although several studies have attempted to examine the underlying mechanisms regarding the effects of DD on $\mathrm{MC}$, a general consensus is not yet reached in the literature.

Initial studies suggested that MCs became unresponsive after $\mathrm{DD}$ as the consequence of internalization of FceRI through progressive cross-linking at low antigen concentrations (Shalit and Levi-Schaffer, 1995; Morales et al., 2005). More recent studies have shown that antigen/IgE/FceRI may remain on the surface during DD (Gladys et al., 2016). In particular, it has been shown that surface IgE was not completely internalized, leaving enough IgE bound on the cell surface to bind Ag and potentially cause degranulation.

It would seem that MCs' hypo-responsiveness is attributable, at least partially, to the abrogation of $\mathrm{Ca} 2+$ mobilization, a critical determinant of degranulation and cytokine production in mast cells (Gladys et al., 2016). Specifically, the organization and spatial distribution of actin is critical for $\mathrm{Ca} 2+$ mobilization in several cell types, including MCs, as demonstrated by in vitro findings and in vivo mouse models of rapid desensitization.

Of note, MRGPRX2-related MC degranulation are probably to DD preocedure as MRGPRX2 receptor does not undergo internalization.

\section{OTHER MECHANISMS INVOLVED IN DRUG DESENSITIZATION: NOT ONLY MAST CELLS}

It is currently assumed that drug tolerance induced by DD is not a permanent state and that it is sustained by a pharmacologic, and not immunologic, tolerance. However, some clinical observations may suggest the induction of a longer memory of tolerance by DD.

Firstly, the rate of reactions during DD procedures progressively reduces over increasing number of desensitisations. As shown by Sloane et al., the percentage of patients with any breakthrough reaction during the DD procedures to chemotherapy and monoclonal antibodies decreased in time with a corresponding increase in the percentage of patients who tolerated the desensitization procedure during repeated cycles (Sloane et al., 2016). Patients initially presenting with anaphylaxis and desensitized in the intensive care setting proceeded to repeated successful desensitisations in the outpatient setting.

Secondly, although protocols are mostly empirical and the best and safest protocol is unknown, it has been reported that after two successful DD cycles, patients may tolerate even shorter subsequent protocols (Sloane et al., 2016).

Finally, DD is widely used in the management of immediate reactions, whereas in non immediate reactions where a $\mathrm{T}$ cellmediated mechanism predominates the role of $\mathrm{DD}$ is still limited. However, in mild clinical conditions such as maculapapular exanthemas and fixed drug eruptions, some DD protocols are successfully applied (Castells et al., 2012; Scherer et al., 2013).
Overall, these observations raise the suspicion that other cells and mechanisms are involved in DD.

\section{Modulation of Adaptive Immune Response to the Drug During DD}

The adaptive immune response sustained by drug-specific $T$ cells and its modification during DD procedures has been scarcely evaluated until now. One study, focused on aspirin DD in patients suffering from aspirin-exacerbated respiratory disease (AERD), showed that one month after beginning of DD, no difference was detectable in the percentage of $\mathrm{CD}^{+} \mathrm{T}$ cells and their cytokine production (IL-2, IL-4 and IFN-g) in comparison with baseline (Atkas et al., 2013). However, the lack of effects on $\mathrm{T}$ cells described do not exclude long-term effects of DD.

Concerning other immediate hypersensitivity reactions (HRs), such as those induced by biological agents (BA), we have recently shown that drug-specific $\mathrm{T}$ cell proliferation to infliximab (IFX) was progressively reduced during DD procedures in a patient suffering from allergic asthma with grass sensitization who had experienced an IFX-induced anaphylaxis. Accordingly, the humoral response to the drug (anti-IFX antibodies titer) showed a parallel decrease over successful DD cycles (Vultaggio et al., 2020). These DD-induced modifications of both cellular and humoral response to IFX were drug-specific, as anti-grass pollen $\operatorname{IgE}$ remained positive during the entire protocol as well as the cellular response to $\mathrm{Phl}$ p5 was consistently positive in all tested samples over DD cycles.

Some data are emerging about the modifications of immune response toward biological agents (BA) during DD in patients with delayed reactions. Teraki and Shiohara have shown a decrease in the percentage of $\mathrm{CD} 8+\mathrm{T}$ cells infiltrating the lesion in allopurinol fixed drug eruptions during DD procedure (Teraki and Shiohara, 2004). Overall, these studies provide limited and controversial information, not allowing any significant understanding of the cellular immune mechanisms operating during DD.

\section{Drug Desensitization Increases Regulatory Cytokines}

The impairment of effector responses observed during DD procedures suggested the involvement of regulatory mechanisms operating in successful DD, in a similar way to what happens during allergen immunotherapy. The effects of DD on regulatory cytokine levels in patients desensitized have been evaluated in both immediate and delayed drug hypersensitivity reactions. Gelincik and coworkers have described a significant increase in IL-10 serum levels $24 \mathrm{~h}$ within the end of DD procedures in 24 patients who underwent successful DD for several oral or parental culprit drugs. In the same case series, no changes of IL-4, IL-5 and IFN- $\gamma$ levels were observed. The authors observed a greater increase in IL-10 levels in patients desensitized for chemotherapeutic drugs (Gelincik et al., 2019). An additional study focused on platinum desensitized patients for immediate HRs has confirmed the increase of IL-10 serum levels after DD, with a tendency to reach higher levels of IL-10 after 
multiple cycles (Tüzer et al. 2020). Regarding delayed HRs, in a case report about DD to allopurinol after a fixed drug eruption, an increase of IL-10 (and IL-6) production by peripheral blood mononuclear cells, was observed. Intracellular IL-10 contents in T cells, but not serum levels, have been analyzed in one study involving patients during desensitization to aspirin in AERD. A decrease of IL-10 (and IFN- $\gamma$ ) intracellular expression in $\mathrm{CD}^{+}$ T cells, was observed after 1 month of desensitization (Aksu et al, 2014). This discrepancy may be caused by the type of reaction where DD has been applied (pathogenesis of AERD) and by the fact that serum levels were not evaluated in this study.

IL-10 is an important regulatory and anti-inflammatory cytokine, largely studied and involved in successful allergen immunotherapy (AIT) (Ni et al., 2015). Studies involving AIT showed the role for IL-10 production by T cells (Treg) and B cells (Breg) in inducing tolerance (Akdis et al., 2005, van de Veen, 2017). It important to note that before Treg and Breg cells appear, early desensitization effect of AIT seems to be associated with IL10 produced by other cells, such as basophils and MCs (van de Veen et al., 2017). In fact, different cells may produce IL-10, such as cells of adaptive ( $\mathrm{T}$ cells, B cells) and innate immunity (dendritic cells, natural killer $\mathrm{T}$ cells, eosinophils, neutrophils, basophils, MC) and keratinocytes (Saraiva and O'Garra et al., 2010). The cellular source of IL-10 production during DD has to be defined as yet, and further studies are required in this field, however we might speculate that the immunological effects of DD are similar to those observed during AIT.

IL-35 is the newest member of IL-12 family. It is a dimeric protein consisting of two separate subunits, an IL-12 subunit $\alpha$ chain (P35) and IL-27 subunit Epstein-Barr virus-induced gene 3 (EBI3) $\beta$ chain; IL-35 has manifested suppressive actions on the immune system. It is secreted by a variety of cells, and then activates its receptors through JAK/STAT signaling to exert its antiinflammatory and immunosuppressive effects (Zhang, et al., 2019). In a patient desensitized to IFX, serum IL-35 was highly increased after each DD cycle and a progressive increase of baseline values in serum samples collected before each cycle was observed (Vultaggio et al., 2020). Such response to high antigen dose during DD is likely comparable to that described for AIT in which IL-35 has been recently described to play a relevant role (Shamji and Durham, 2017; Shamji et al., 2018) and confirm the involvement of regulatory cytokines in the DD-related immunological mechanisms.

\section{Drug Desensitization Induces Drug-SpecificTreg Cells}

Regulatory $\mathrm{T}$ (Treg) cells are a subset of $\mathrm{CD} 4^{+} \alpha \beta \mathrm{T}$ cells that play a major role for maintaining self tolerance and preventing autoimmunity, limiting chronic inflammatory diseases, dampening homeostatic lymphocyte expansion, and suppressing immune responses to parasites and viruses and tumors, including that induced by therapeutic vaccines. The manipulation of Treg functions is an important goal of AIT, since a successful AIT is sustained by the generation of allergenspecific Tregcells (Palomares et al., 2010), and, as recently shown, in particular by $\mathrm{T}$ cells producing the regulatory cytokine IL-35 (called Tr35) (Shamji et al. 2018).
The involvement of Treg cells in DD mechanisms has been analyzed in few studies until now, mainly regarding DD after HRs to monoclonal antibodies. An increase of $\mathrm{CD} 4{ }^{+} \mathrm{CD} 25^{+}$cells and $\mathrm{CD} 4{ }^{+} \mathrm{CD} 25$ + FoxP3 + Treg cells in peripheral blood after DD for rituximab has been described in a successful procedure in a patient suffering from nephrotic syndrome (Aydogan et al., 2013). In addition, during DD for IFX, PBMC upon in vitro stimulation with IFX were able to produce IL-35 in a MHC-Class II-restricted manner, suggesting that the production of this regulatory cytokine is sustained by the presence of drugspecific Tr35. Notably these cells constitutively express check point molecules, including PD1 (Turnis et al., 2016), and accordingly, in the same case report, increased proportion of circulating $\mathrm{CD}^{+} \mathrm{CD} 4+\mathrm{PD} 1+$ and $\mathrm{CD}^{+} \mathrm{CD} 4+\mathrm{Foxp} 3+\mathrm{T}$ cells were observed after the second and third cycle of desensitization. Regarding delayed reactions induced by allopurinol, Teraki and Shiohara showed that the number of $\mathrm{CD} 4{ }^{+} \mathrm{CD} 25^{+} \mathrm{T}$ cells increased in skin lesions after the beginning of DD, suggesting that Treg cells may migrate from blood to skin, where they might act to suppress effector T cells, that conversely decreased in proportion (from 91\% to 35\%) (Teraki and Shiohara, 2004). Even though these data obtained in single case reports must be considered preliminary, overall, they suggest a possible role of Tregulatory cells in the drug tolerance induced by DD.

\section{DISCUSSION}

Drug desensitization allows a transient clinical tolerance to the culprit drug by administering, in a short time, increasing amounts of the drug until reaching the therapeutic dose. Such procedure is to be applied mainly in reactive patients with no alternative treatment options.

Besides a profound change of MC reactivity with inhibition of their activation pathways and mediators' release (only partially known until now), some reports strongly indicate that tolerance induced by DD implies the modulation of drug-specific response by regulatory mechanisms, confirming that this type of procedure deeply impacts on the immune response, more than that has been demonstrated to date. Of note, this regulatory activity is transient and lasts a short and variable period after stopping the treatment.

Drug-specific immune response to BAs is down-regulated by a panel of regulatory cytokines, including the traditional IL-10 and a new molecule belonging to IL-12 family, IL-35. In particular, the activation/expansion of drug-specific Tr35 cells, occurring during the DD procedure to some BAs, may have a particular relevance in the mechanisms of DD tolerance, since IL-35 orchestrates other regulatory cells and cytokines. Figure 1 illustrates the immunological modifications of both umoral and cellular adaptive immune response to BA during DD.

It would be desirable to establish in the near future which regulatory cells (Treg, Breg, ILCreg, DCreg etc) are involved in each drug treatment, when they become operative during the DD procedure, and how long they last after stopping the DD. In addition, we cannot exclude that the type of drug, the route of administration, the dose and the scheme could influence the mechanisms operating in successful DD. 

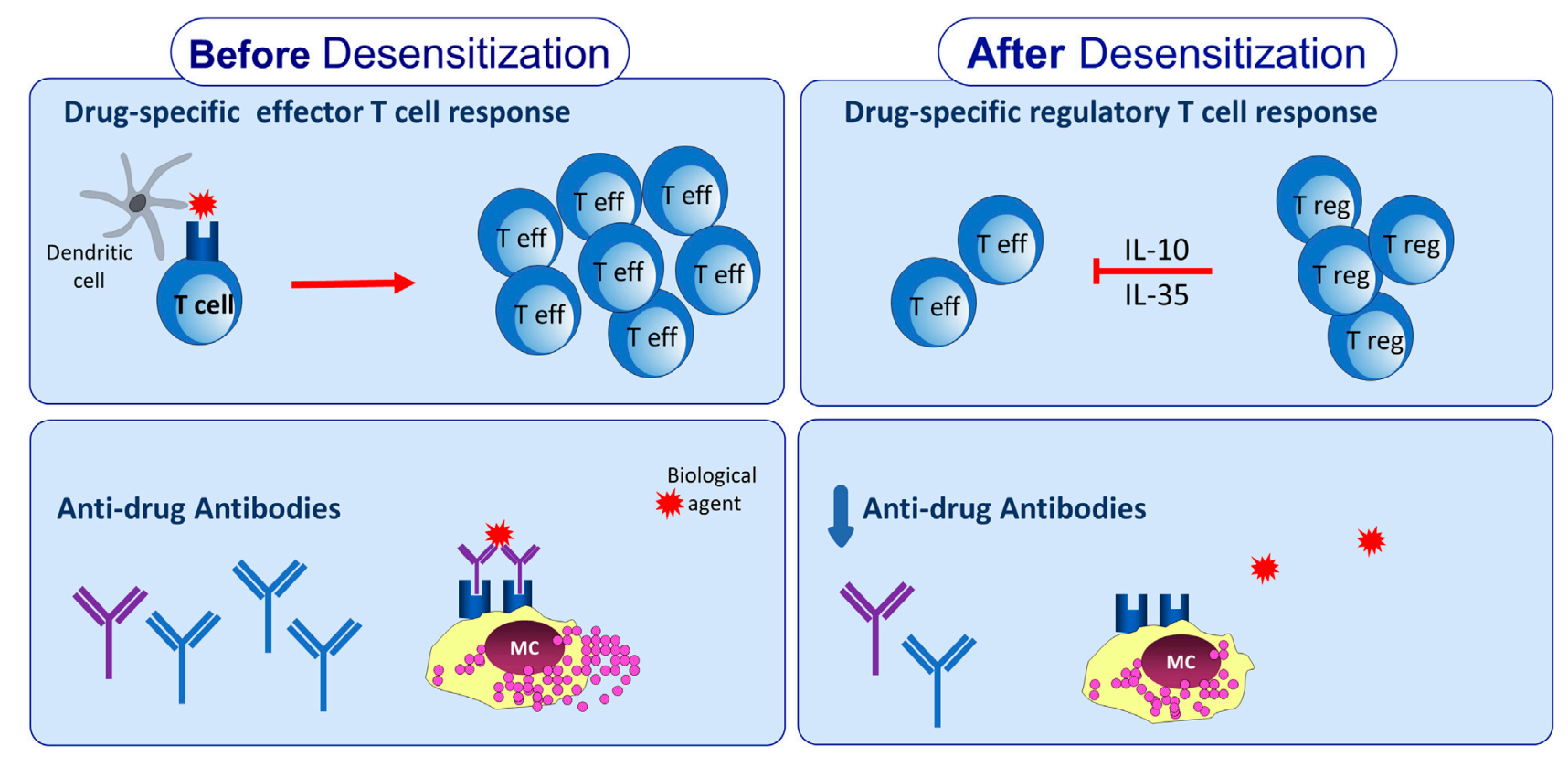

Figure 1 | Adaptive immunological changes induced by drug desensitization. Drug desensitization may impact on adaptive immune response leading to reduction of anti-drug antibodies levels. In addition, drug-specific $\mathrm{T}$ cell response seems to be affected by drug desensitization, due to expansion of $\mathrm{T}$ regulatory cells able to produce IL-10 and IL-35.

In conclusion, DD procedure induces two independent antigen-specific mechanisms: the release of controlled cellular mediators by $\mathrm{MC}$ over time and the development of the antigenspecific regulation of adaptive response. These mechanisms allow to safely and successfully reach the target dose of the drug.

\section{REFERENCES}

Akdis, M., Blaser, K., and Akdis, C. A. (2005). T regulatory cells in allergy: novel concepts in the pathogenesis, prevention, and treatment of allergic diseases. J. Allergy Clin. Immunol. 116, 961-969. doi:10.1016/j.jaci.2005.09.004

Aksu, K., Kurt, E., Alatas, Ö., and Gülbas, Z. (2014). Effect of aspirin desensitization on T-cell cytokines and plasma lipoxins in aspirin-exacerbated respiratory disease. Allergy Asthma Proc. 35, 148-155. doi:10.2500/aap.2014.35.3726

Aktas, A., Kurt, E., and Gulbas, Z. (2013). Cytokine expression before and after aspirin desensitization therapy in aspirin-exacerbated respiratory disease. Inflammation 36, 1553-1559. doi:10.1007/s10753-013-9699-7

Aydogan, M., Yologlu, N., Gacar, G., Uyan, Z. S., Eser, I., and Karaoz, E. (2013). Successful rapid rituximab desensitization in an adolescent patient with nephrotic syndrome: increase in number of Treg cells after desensitization. J. Allergy Clin. Immunol. 132, 478-480. doi:10.1016/j.jaci.2013.02.004

Bonamichi-Santos, R., and Castells, M. (2016). Diagnoses and management of drug hypersensitivity and anaphylaxis in cancer and chronic inflammatory diseases: reactions to taxanes and monoclonal antibodies. Clin. Rev. Allergy Immunol. 54, 375-385. doi:10.1007/s12016-016-8556-5

Boyce, J. A. (2019). Aspirin sensitivity: lessons in the regulation (and dysregulation) of mast cell function. J. Allergy Clin. Immunol. 144, 875-881. doi:10.1016/j.jaci. 2019.08 .022

Castells, M. (2017). Drug hypersensitivity and anaphylaxis in cancer and chronic inflammatory diseases: the role of desensitizations. Front. Immunol. 8, 1472. doi:10.3389/fimmu.2017.01472

Castells, M., Sancho-Serradel, M. C., and Simarro, M. (2012). Hypersensitivity to antineoplastic agents: mechanisms and treatment with rapid desensitization. Cancer Immunol. Immunother. 61, 1575-1584. doi:10.1007/s00262-012-1273-x

\section{AUTHOR CONTRIBUTIONS}

$\mathrm{AV}$ and $\mathrm{AM}$ wrote the manuscript. FN designed the figure. EM, $\mathrm{SB}$ and $\mathrm{EV}$ revised the manuscript. All authors contributed to the article and approved the submitted version.

Castells, M. C. (2015). A new era for drug desensitizations. J. Allergy Clin. Immunol. Pract. 3, 639-640. doi:10.1016/j.jaip.2015.05.006

Castells, M. C. (2017). Diagnosis and management of anaphylaxis in precision medicine. J. Allergy Clin. Immunol. 140, 321. doi:10.1016/j.jaci.2017.06.011 doi:10.1016/j.jaci.2017.06.012

De las Vecillas Sánchez, L., Alenazy, L., Garcia-Neuer, M., and Castells, M. (2017). Drug hypersensitivity and desensitizations: mechanisms and new approaches. Int. J. Mol. Sci. 18, 1316. doi:10.3390/ijms 18061316

Gelincik, A., Demir, S., Şen, F., Bozbey, U. H., Olgaç, M., Ünal, D., et al. (2019). Interleukin-10 is increased in successful drug desensitization regardless of the hypersensitivity reaction type. Asia Pac Allergy 28, 9. doi:10.5415/apallergy. 2019.9.e9.

Gladys, A. W. X., Church, A. M., Kulis, M., Choi, H. W., Abraham, S. N., and Abraham, S. N. 2016). Mast cell desensitization inhibits calcium flux and aberrantly remodels actin. J. Clin. Invest. 126, 4103-4118. doi:10.1172/JCI87492

Guvenir, H., Dibek Misirlioglu, E., Capanoglu, M., Buyuktiryaki, B., Unal, O., Toyran, M., et al. (2017). Successful desensitization of elosulfase alfa-induced anaphylaxis in a pediatric patient with Morquio syndrome. J. Allergy Clin. Immunol. Pract. 5, 1156-1157. doi:10.1016/j.jaip.2017.02.020

Jimenez-Rodriguez, T., Garcia-Neuer, M., Alenazy, L. A., and Castells, M. (2018). Anaphylaxis in the 21st century: phenotypes, endotypes, and biomarkers. J. Asthma Allergy 11, 121-142. doi:10.2147/jaa.s159411

Lee, C. W., Matulonis, U. A., and Castells, M. C. (2004). Carboplatin hypersensitivity: a 6-h 12-step protocol effective in 35 desensitizations in patients with gynecological malignancies and mast cell/IgE-mediated reactions. Gynecol. Oncol. 95, 370-376. doi:10.1016/j.ygyno.2004.08.002

Madrigal-Burgaleta, R., Bernal-Rubio, L., Berges-Gimeno, M. P., Carpio-Escalona, L. V., Gehlhaar, P., and Alvarez-Cuesta, E. (2019). A large single hospital experience using drug provocation testing and rapid drug desensitization in 
hypersensitivity to antineoplastic and biological agents. J. Allergy Clin. Immol. Pract. 7, 618-632. doi:10.1016/j.jaip.2018.07.031

McNeil, B. D., Pundir, P., Meeker, S., Han, L., Undem, B. J., Kulka, M., , et al.Dong, X. (2015). Identification of a mast-cell-specific receptor crucial for pseudoallergic drug reactions. Nature 519, 237-241. doi:10.1038/nature14022

Mishra, S., Connors, L., and Tugwell, B. (2018). Role of omalizumab in insulin hypersensitivity: a case report and review of the literature. Diabet. Med. 35, 663-666. doi:10.1111/dme.13591

Morales, A. R., Shah, N., and Castells, M. (2005). Antigen-IgE desensitization in signal transducer and activator of transcription 6-deficient mast cells by suboptimal doses of antigen. Ann. Allergy Asthma Immunol. 94, 575-580. doi:10.1016/s1081-1206(10)61136-2

Nam, J. H., and Kim, W. K. (2020). The role of TRP channels in allergic inflammation and its clinical relevance. Curr. Med. Chem. 27, 1446-1468. doi:10.2174/0929867326666181126113015

Ni, G., Wang, T., Walton, S., Zhu, B., Chen, S., Wu, X., et al. (2015). Manipulating IL-10 signalling blockade for better immunotherapy. Cell. Immunol. 293, 126-129. doi:10.1016/j.cellimm.2014.12.012

Oude Elberink, H. N. G., Jalving, M., Dijkstra, H., and van de Ven, A. A. J. M. (2020). Modified protocol of omalizumab treatment to prevent carboplatininduced drug hypersensitivity reactions: a case study. Clin. Transl. Allergy 10 (5). doi:10.1186/s13601-020-0309-0

Palomares, O., Yaman, G., Azkur, A. K., Akkoc, T., Akdis, M., and Akdis, C. A. (2010). Role of Treg in immune regulation of allergic diseases. Eur. J. Immunol. 40, 1232-1240. doi:10.1002/eji.200940045

Phong, B. L., Avery, L., Sumpter, T. L., Gorman, J. V., Watkins, S. C., Colgan, J. D., et al. (2015). Tim-3 enhances FceRI-proximal signaling to modulate mast cell activation. J. Exp. Med. 212, 2289-2304. doi:10.1084/jem.20150388

Prieto-García, A., Noguerado, B., Rojas, P., Torrado, I., Rodríguez-Fernández, A., and Tornero, P. (2019). Unexpected anaphylaxis after completing a desensitization protocol to oxaliplatin: successful adjuvant use of omalizumab. J Investig. Allergol. Clin. Immunol. 29, 53-55. doi:10.18176/jiaci.0326

Pyle, R. C., Butterfield, J. H., Volcheck, G. W., Podjasek, J. C., Rank, M. A., Li, J. T., et al. (2014). Successful outpatient graded administration of trimethoprimsulfamethoxazole in patients without HIV and with a history of sulfonamide adverse drug reaction. J. Allergy Clin. Immunol. Pract. 2, 52-58. doi:10.1016/j. jaip.2013.11.002

Sala-Cunill, A., Luengo, O., and Cardona, V. (2019). Biologics and anaphylaxis. Curr. Opin. Allergy Clin. Immunol. 19, 439-446. doi:10.1097/aci. 0000000000000550

Sancho-Serra, M.. C., Simarro, M., and Castells, M. (2011). Rapid IgE desensitization is antigen specific and impairs early and late mast cell responses targeting FceRI internalization. Eur. J. Immunol. 41, 1004-1013. doi:10.1002/eji.201040810

Saraiva, M., and O'Garra, A. (2010). The regulation of IL-10 production by immune cells. Nat. Rev. Immunol. 10, 170-181. doi:10.1038/nri2711

Scherer, K., Brockow, K., Aberer, W., Gooi, J. H., Demoly, P., Romano, A., et al. (2013). Desensitization in delayed drug hypersensitivity reactions-an EAACI position paper of the Drug Allergy Interest Group. Allergy 68, 844-852. doi:10. 1111/all.12161

Shalit, M., and Levi-Schaffer, F. (1995). Challenge of mast cells with increasing amounts of antigen induces desensitization. Clin. Exp. Allergy 25, 896-902. doi:10.1111/j.1365-2222.1995.tb00033.x

Shamji, M. H., and Durham, S. R. (2017). Mechanisms of allergen immunotherapy for inhaled allergens and predictive biomarkers. J. Allergy Clin. Immunol. 140, 1485-1498. doi:10.1016/j.jaci.2017.10.010
Shamji, M. H., Kappen, J., Abubakar-Waziri, H., Zhang, J., Steveling, E., Watchman, S., et al. (2018). Nasal allergen-neutralizing IgG. J. Allergy Clin. Immunol. 143, 1067-1076. doi:10.1016/j.jaci.2018.09.039

Sloane, D., Govindarajulu, U., Harrow-Mortelliti, J., Barry, W., Hsu, F. I., Hong, D., et al. (2016). Safety, costs, and efficacy of rapid drug desensitizations to chemotherapy and monoclonal antibodies. J. Allergy Clin. Immunol. Pract. 4, 497-504. doi:10.1016/j.jaip.2015.12.019

Spoerl, D., Nigolian, H., Czarnetzki, C., and Harr, T. (2017). Reclassifying anaphylaxis to neuromuscular blocking agents based on the presumed patho-mechanism: IgE-mediated, pharmacological adverse reaction or "innate hypersensitivity"?. Int. J. Mol. Sci. 18, 1223. doi:10.3390/ijms18061223

Teraki, Y., and Shiohara, T. (2004). Successful desensitization to fixed drug eruption: the presence of $\mathrm{CD} 25+\mathrm{CD} 4+\mathrm{T}$ cells in the epidermis of fixed drug eruption lesions may Be involved in the induction of desensitization. Dermatology 209, 29-32. doi:10.1159/000078583

Turnis, M. E., Sawant, D. V., Szymczak-Workman, A. L., Andrews, L. P., Delgoffe, G. M., Yano, H., et al. (2016). Interleukin-35 limits anti-tumor immunity. Immunity 44, 316-329. doi:10.1016/j.immuni.2016.01.013

Tüzer, C., Sari, M., AktaşÇetin, E., Ak, N., Büyüköztürk, S., Çolakoğlu, B., et al. (2020). Rapid drug desensitization for platinum-based chemotherapy drugs significantly increases peripheral blood IL-10 levels. Allergy. doi:10.1111/all.14311

Van de Veen, W. (2017). The role of regulatory B cells in allergen immunotherapy. Curr Opin. Allergy Clin. Immunol. 17, 447-452. doi:10.1097/ACI.0000000000000400

Van de Veen, W., Wirz, O. F., Globinska, A., and Akdis, M. (2017). Novel mechanisms in immune tolerance to allergens during natural allergen exposure and allergen-specific immunotherapy. Curr. Opin. Immunol. 48, 74-81. doi:10.1016/j.coi.2017.08.012

Vultaggio, A., and Castells, M. C. (2014). Hypersensitivity reactions to biologic agents. Immunol. Allergy Clin. 34, 615-632. doi:10.1016/j.iac.2014.04.008

Vultaggio, A., Maggi, E., and Matucci, A. (2011). Immediate adverse reactions to biologicals: from pathogenic mechanisms to prophylactic management. Curr. Opin. Allergy Clin. Immunol. 11, 262-268. doi:10.1097/aci. 0b013e3283464bcd

Vultaggio, A., Nencini, F., Bormioli, S., Dies, L., Vivarelli, E., Maggi, E., et al. (2020). Desensitization modulates humoral and cellular immune response to infliximab in a patient with an immediate hypersensitivity reaction. J. Allergy Clin. Immunol. Pract. 8, 1764-1767. doi:10.1016/j.jaip.2019.12.040

Waldram, J., Walters, K., Simon, R., Woessner, K., Waalen, J., and White, A. (2018). Safety and outcomes of aspirin desensitization for aspirin exacerbated respiratory disease: a single center study. J. Allergy Clin. Immunol. 141, 250-256. doi:10.1016/j.jaci.2017.05.006

Zhang, J., Zhang, Y., Wang, Q., Li, C., Deng, H., Si, C., et al. (2019). Interleukin 35 in immune-related diseases: protection, or destruction. Immunology 157, 13-20. doi:10.1111/imm.13044

Conflict of Interest: The authors declare that the research was conducted in the absence of any commercial or financial relationships that could be construed as a potential conflict of interest.

Copyright $\odot 2020$ Vultaggio, Matucci, Nencini, Bormioli, Maggi and Vivarelli. This is an open-access article distributed under the terms of the Creative Commons Attribution License (CC BY). The use, distribution or reproduction in other forums is permitted, provided the original author(s) and the copyright owner(s) are credited and that the original publication in this journal is cited, in accordance with accepted academic practice. No use, distribution or reproduction is permitted which does not comply with these terms. 